\title{
硅卡宾铁(0)氮气配合物催化的炔烃选择性硼氢化反应
}

\author{
白云平熣春明* \\ (南开大学元素有机化学国家重点实验室及化学学院 天津 300071)
}

\begin{abstract}
摘要 硅卡宾 $\left(\mathrm{R}_{2} \mathrm{Si}\right.$ :, silylene) 是卡宾的相似体, 可以作为配体与金属形成配合物. 由于硅的原子半径比碳大, 硅卡宾可 与 Lewis 碱配位形成三配位甚至四配位的化合物同时保持很强的配位能力. 因此, 硅卡宾兼具卡宾和膦配体的结构特 征，在稳定新颖的金属配合物及均相催化领域或具有更大的调控空间。本工作报道硅卡宾铁氮气配合物 $\left[\mathrm{PhC}(t-\mathrm{BuN})_{2} \mathrm{SiCH}_{2} \mathrm{C}(t-\mathrm{Bu}) \mathrm{NAr}\right] \mathrm{FeN}_{2}\left(\mathbf{D}, \mathrm{Ar}=2,6-(i-\mathrm{Pr})_{2} \mathrm{C}_{6} \mathrm{H}_{3}\right)$ 催化的炔烃的选择性嗍氢化反应. 研究发现, 该配合物对 炔烃的硼氢化反应具有很好的区域及立体选择性, 主要生成 $E$ 式构型产物并表现出很好的官能团耐受性. 该研究表明, 硅卡宾对过渡金属催化具有很好的调控作用，具有很好的研究潜力.
\end{abstract}

关键词 硅卡宾; 铁催化; 硼氢化; 炔烃; 氮气

\section{Selective Hydroboration of Alkynes Enabled by a Silylene Iron(0) Dinitrogen Complex}

\author{
Bai, Yunping Cui, Chunming* \\ (State Key Laboratory of Elemento-organic Chemistry and College of Chemistry, Nankai University, Tianjin 300071, China)
}

\begin{abstract}
Silylenes, isoelectronic with carbenes, are a kind of key intermediates in organosilicon chemistry. They possess a lone pair and an empty orbital on the silicon center, and thus could be used as donors and acceptors. Consequently, they could form complexes with various metals to support new structures and chemistry similar to both carbenes and phosphines. Iron complexes played important roles in the development of catalysts because of the inexpensive, nontoxic and sustainable characteristics. Catalytic hydroboration of alkynes presents the most atom-economic and straightforward protocol for the synthesis of vinylboranes which are indispensable intermediates for $\mathrm{C}-\mathrm{C}$ coupling reactions. For the catalytic hydroboration of alkynes with iron catalysts, Enthaler's group developed the first iron catalytic system for hydroboration of alkynes by using $\mathrm{Fe}_{2}(\mathrm{CO})_{9}(\mathrm{~A}$, Chart 1) as the catalyst. Almost at the same time, Thomas's group reported the bis(imino)pyridine derived iron complexes (B) in combination with an activator for catalytic hydroboration of alkynes and alkenes. In 2017, Nishibayashi and co-workers employed an iron(II) hydride complex (C) supported by a PNP pincer ligand for catalytic $E$-selective hydroboration of alkynes. In 2020, Findlater et al. reported the regioselective hydroboration of alkynes and alkenes with iron complexes supported by bis(2,6-diisopropylaniline)acenaphthene ligands. However, these catalysts still suffered from limited substrate scope or harsh conditions. The development of highly selective catalysts for a wide substrate scope is still desirable. On the basis of our design on silylene ligands for iron chemistry, we are interested in the silylene-iron complexes for catalytic hydroboration reactions. In this paper, hydroborylation of terminal alkynes catalyzed by a neutral silylene-imine iron(0) dinitrogen complex $\mathbf{D}$ was studied. The reaction is highly regio- and stereoselective and almost exclusively gave $E$-hydroboration products. The optimized reaction conditions are as following: To a dried Schlenk tube were added complex D $(0.006 \mathrm{~g}, 0.01 \mathrm{mmol})$, toluene $(1.0 \mathrm{~mL})$, alkyne $(0.20 \mathrm{mmol})$, and catechol borane $(0.02 \mathrm{~g}, 0.20 \mathrm{mmol})$. After the mixture was stirred at $80{ }^{\circ} \mathrm{C}$ for $24 \mathrm{~h}$, it was cooled down to room temperature. The solvents were removed under vacuum and the residue was purified by flash chromatography on silica gel to afford the desired products.

Keywords silylene; iron-catalyzed; hydroboration; alkyne; dinitrogen
\end{abstract}

\section{1 引言}

硅卡宾 $\left(\mathrm{R}_{2} \mathrm{Si}\right.$ :, silylene) 是卡宾的同族类似物, 它具 有空轨道和孤对电子而表现出 Lewis 酸性和碱性. 与卡 宾不同的是, 硅卡宾的基态为单线态, Lewis 酸性很强 导致其在常规条件下非常不稳定 ${ }^{[1]}$. 近年来研究发现 ${ }^{[2]}$,
硅卡宾可以通过与 Lewis 碱配位形成相对稳定的化合物 $\left(\mathrm{R}_{2} \mathrm{Si} \leftarrow\right.$ Donor), 并表现出很强的给电子能力及亲核性, 因此, 硅卡宾化学近年来得到了迅速发展. 虽然硅卡宾 的金属配合物已有不少报道 ${ }^{[2,3]}$, 但与卡宾及膦配体配 合物相比, 其类型及数目仍然非常有限, 特别是用于调 控催化反应的报道非常少见 ${ }^{[3-6]}$. 硅是地壳中最丰富的

*E-mail: cmcui@nankai.edu.cn; Tel.: +86-22-23503461; Fax: +86-22-23503461.

Received May 12, 2020; published June 28, 2020.

Supporting information for this article is available free of charge via the Internet at http://sioc-journal.cn.

Project supported by the National Natural Science Foundation of China (No. 21632006).

项目受国家自然科学基金(No. 21632006)资助. 
元素之一，同时有机硅化合物通常具有很好的生物相容 性, 因此, 发展有机硅的配合物化学对可持续化学及发 展新颖的化学转化无疑具有重要的价值.

有机硼是重要的 $\mathrm{C}-\mathrm{C}$ 偶联中间体, 烯烃及炔烃的 嗍氢化反应是制备有机硼最直接的方法 ${ }^{[7]}$. 通过金属催 化的方法可以很好地调控反应的区域及立体选择性, 因 此, 催化硼氢化反应已成为选择性合成有机硼化合物最 高效及原子经济性合成方法, 受到大家广泛关注. 近年 来, 铁催化的硼氢化反应也备受关注. 2013 年, Enthaler 等 ${ }^{[8]}$ 报道了 $\mathrm{Fe}_{2}(\mathrm{CO})_{9}(\mathbf{A})$ 催化的炔烃硼氢化反应, 主要生 成 $E$ 式构型的产物. 同年, Thomas 团队 ${ }^{[9]}$ 利用吡啶二亚 胺配体及其衍生物与铁形成的络合物(B)在活化剂的存 在下催化炔烃及烯烃的硼氢化反应。2017 年, Nishibayashi 团队 ${ }^{[10]}$ 报道了吡咯双膦螯合型 PNP 配体的 铁氢络合物 $(\mathbf{C})$ 催化的末端炔烃嗍氢化反应, 同样生成 $E$ 式构型的产物. 2020 年, Findlater 团队 ${ }^{[11]}$ 利用含 BIAN (bis(2,6-diisopropylaniline)acenaphthene)配体的铁配合物 在助催化剂的存在下, 可高效地催化炔烃及烯烃的嘲氢 化反应.

我们在前期研究发现, 有机硅卡宾配体可以稳定铁 的氮气配合物 $\mathbf{D}($ Chart 1), 并可以很好调控铁的氧化态 变化. 进一步研究发现, D 可以催化氮气的硅化反应, 表现出较高的催化活性 ${ }^{[12]}$. 为了进一步研究该配合物 的功能, 我们研究了 $\mathbf{D}$ 在催化炔烃嗍氢化反应中的应 用, 发现该铁配合物对硼氢化反应具有很好的调控能 力.

\section{Chart 1}

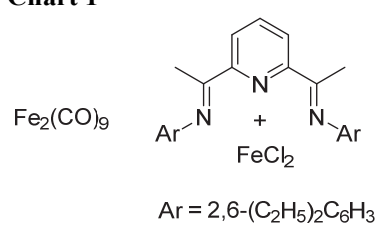

A

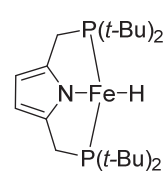

C

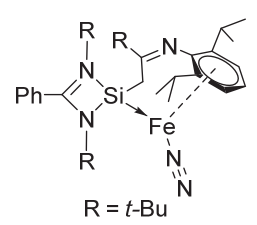

D
This work:

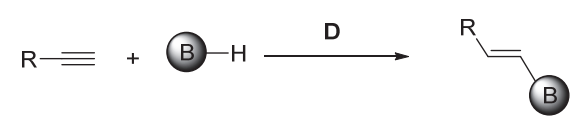

\section{2 结果与讨论}

\section{1 反应条件的优化}

我们以苯乙炔的硼氢化反应作为模板, 对催化反应 的条件进行了优化. 首先以配合物 $\mathbf{D}$ 为催化剂, 在 $n_{\mathbf{D}}$ / $n_{\text {苯乙炔 }}=5 \%$ 的情况下, 对反应温度, 嗍化试剂及溶剂等条 件进行了优化(表 1), 反应进程可通过 ${ }^{1} \mathrm{H}$ NMR 及 GC-MS 进行监测. 从表 1 可以看出, 反应在常温条件下
(Entries 5 and 6)收率很低，当温度升到 $80{ }^{\circ} \mathrm{C}$ 时，可以 达到几乎定量的收率(Entries 1 and 7). 溶剂对反应也有 很大的影响, 反应在甲苯中进行明显优于在四氢呋喃及 正己烷中(Entries 1 3). 值得注意的是, 配合物 $\mathbf{D}$ 在氮 气氛围中催化活性及选择性均比在氩气氛围中更好 (Entries 1 与 4), 这很有可能是含有氮气配体的催化剂 $\mathbf{D}$ 在氮气气氛下相对比较稳定的缘故. 当用儿茶酚硼烷 (HBCat)代替频哪醇嗍烷(HBPin)作硼氢化试剂时, 该体 系的选择性有所提高(表 1, 比较 Entries 1 与 7 以及 Entries 5 与 6). 但催化剂用量降低时(表 1, 比较 Entries 7 与 8)转化率明显降低. 所有实验表明, 嗍氢化产物以 $E$ 式构型为主, 其 $E / Z$ 比例为最高可达 $97 ： 3$. 基于这些 结果, 我们以 $\mathbf{D}$ 为催化剂, $n_{\mathbf{D}} / n_{\text {苯乙炏 }}=5 \%, 80{ }^{\circ} \mathrm{C}$, 氮气气 氛及甲苯溶剂中, 以 HBCat 为硼化试剂对反应底物进行 了扩展.

表 1 反应条件的优化

Table 1 Optimization of reaction conditions

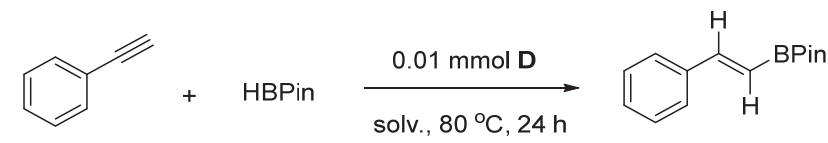

$0.20 \mathrm{mmol}$

$0.20 \mathrm{mmol}$

E

\begin{tabular}{ccccccc}
\hline Entry $^{a}$ & Borane & Solvent & $T /{ }^{\circ} \mathrm{C}$ & Gas & Yield/\% & $E / Z$ \\
\hline 1 & HBPin & toluene & 80 & nitrogen & $>99$ & $95: 5$ \\
2 & HBPin & THF & 80 & nitrogen & 64 & $94: 6$ \\
3 & HBPin & $n$-hexane & 80 & nitrogen & 60 & $93: 7$ \\
4 & HBPin & toluene & 80 & argon & 86 & $93: 7$ \\
5 & HBPin & toluene & 25 & nitrogen & 38 & $85: 15$ \\
6 & HBcat & toluene & 25 & argon & 36 & $95: 5$ \\
7 & HBCat & toluene & 80 & nitrogen & $>99$ & $97: 3$ \\
$8^{b}$ & HBCat & toluene & 80 & nitrogen & 86 & $97: 3$ \\
\hline${ }^{a} 0.20 \mathrm{mmol}$ of alkynes and borane, $1 \mathrm{~mL}$ of solvent. ${ }^{b} 0.005$ mmol of catalyst.
\end{tabular}

\section{2 反应底物的拓展}

我们对不同取代基的末端炔烃进行了研究，如图 1 所示，反应无论是对苯基和芳杂环取代还是烷基取代的 炔烃都显示很好的区域及立体选择性(图 1, a、i、j 和 $\mathbf{k}$ ). 此外, 我们研究了苯环上取代基的电子效应对反应的影 响: 当苯基对位上带有吸电子基团如三氟甲基、氟及酯 基时，反应速率更快，产物选择性更好(图 1, b、c、d); 当 苯环带有给电子基团如甲氧基、甲基时反应转化率明显 降低(图 1, e $\mathbf{f}$ 和 $\mathbf{g}$ ). 但硝基苯取代的炔烃不反应, 这可 能是由于硝基会使催化剂分解而造成的(图 1, h). 另外, 底物位阻增加也对催化反应有明显的抑制 (图 1, g). 该 催化体系对噻吩取代的炔烃显示更好的活性及选择性 (图 1, i); 对于烷基取代炔烃反应活性高, 但产物选择性 稍有降低(图 1, k).

进一步研究发现, 该催化反应不适用于内炔烃, 这 很有可能是由于内炔烃有较大的空间位阻的缘故. 


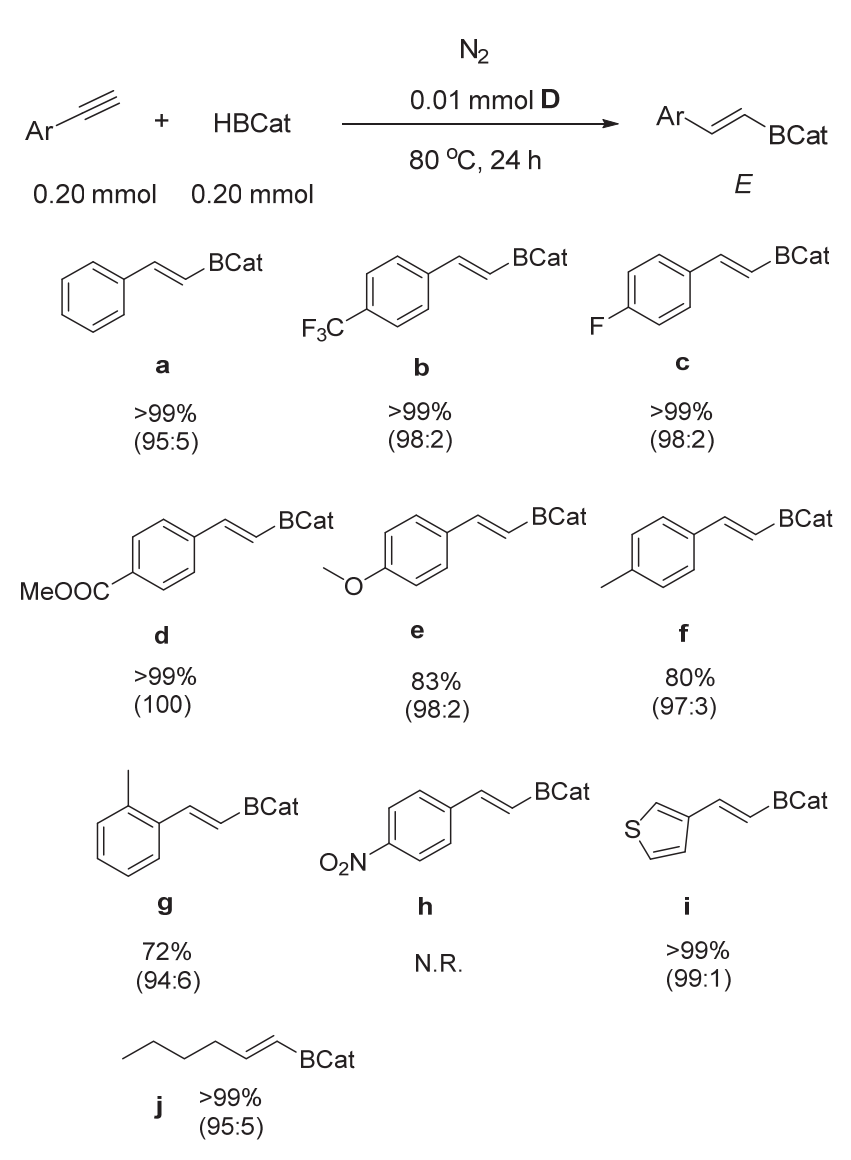

图 1 适用的底物

Figure 1 Substrate scope

\section{3 可能的反应机理}

尽管铁催化的嗍氢化反应有一定数量的报道 ${ }^{[8-11,13]}$, 但对其反应机理一直存在较大的分歧. 相当一部分的铁 催化的硼氢化反应需要额外的强碱来对铁配合物进行 活化, 其目的是原位产生活性低价态铁或铁的氢化

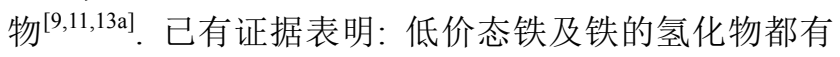
可能是反应的关键中间体，但反应途径不同. 到目前为 止, 推测的低价态铁对 $\mathrm{B}-\mathrm{H}$ 键的氧化加成以及与炔烃 形成配合物的初始机理都有报道. 另一方面, 也有研究 表明: 炔烃对 $\mathrm{Fe}-\mathrm{H}$ 及 $\mathrm{Fe}-\mathrm{B}$ 键的插入反应都有可能是 其催化循环的关键步骤 ${ }^{[13]}$.

我们分别尝试了D与 HBCat及炔烃的反应, 前者反 应复杂, 没能分离和检测到明确的产物. 但与炔烃反应 时发现发生了炔烃的三聚, 形成了多取代苯. 通过对铁 的硅卡宾配合物的电子结构分析, 我们发现硅卡宾具有 很强的接受电子能力, 与 $\mathrm{CO}$ 一样是典型的 $\pi$-酸配体. 因此, 配合物 D 与 $\mathrm{B}-\mathrm{H}$ 键进行氧化加成反应会受到硅 卡宾配体的抑制. 基于以上分析, 我们认为 $\mathbf{D}$ 催化的路 氢化反应的初始步骤很有可能是通过铁中心对炔烃的 活化引发的(图 2). 催化剂 D 首先失去配位的氮气产生 不饱和配合物 $\mathbf{E}$, 继而与炔烃进行配位形成中间体 $\mathbf{F}$. 炔烃由于配位被活化进而与 $\mathrm{B}-\mathrm{H}$ 键进行加成形成产物 脱去, 同时, 再次生成催化剂 E. 由于炔烃配位, 该反
应的区域及立体选择性主要通过空间位阻来进行调控, 嗍原子主要加成在位阻小的末端. 由于硅卡宾-亚胺配 体有很大的位阻，内炔烃很难与 $\mathbf{E}$ 进行配位，因此反应 很难发生.

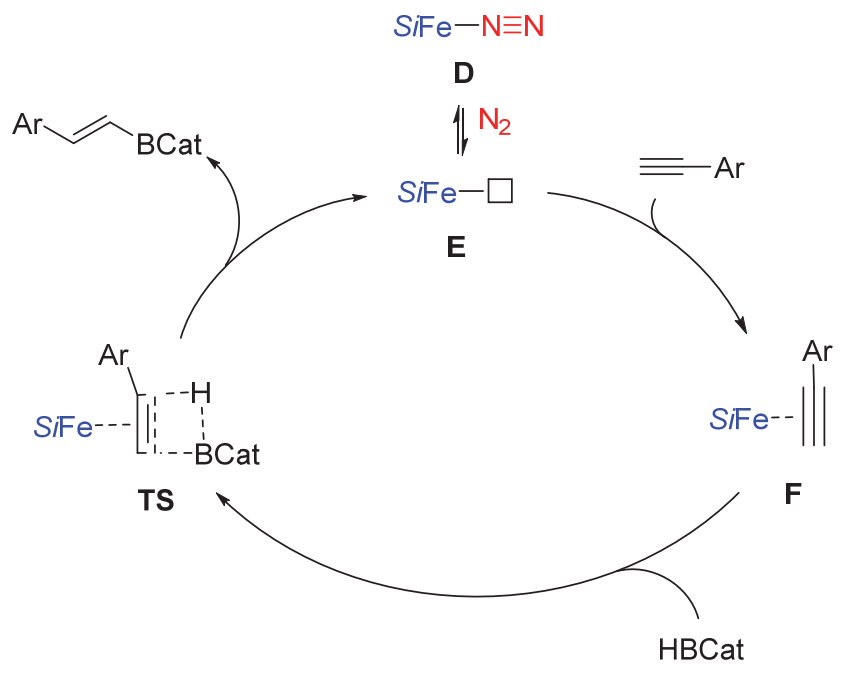

图 2 可能的反应机理

Figure 2 Proposed reaction mechanism

\section{3 结论}

以硅卡宾-亚胺双齿配体与铁形成的氮气配合物为 催化体系, 实现了末端炔烃与儿茶酚嗍烷的选择性硼氢 化反应. 反应产物同时具有较高的收率及 $E$ 式选择性. 与一些已有铁催化剂相比 ${ }^{[9,11,13 a]}$, 该体系无需碱添加剂, 对芳基及烷基取代的末端炔烃显示相同的区域及立体 选择性, 表明空间位阻起主要作用. 下一步我们将进一 步研究硅卡宾配合物与底物的作用本质, 进一步认识硅 卡宾对金属反应性的调控的规律, 发展新的转化过程, 进一步拓展和发展硅卡宾化学.

\section{4 实验部分}

一般反应过程如下：将配合物 D (0.006 g，0.01 $\mathrm{mmol}$ )和甲苯 $(1 \mathrm{~mL})$ 加到 $15 \mathrm{~mL}$ 带磁子的封管中, 形成 溶液. 然后将炔烃 $(0.20 \mathrm{mmol})$ 及儿茶酚硣烷 $(0.02 \mathrm{~g}$, $0.20 \mathrm{mmol}$ )依次加入体系中. $80{ }^{\circ} \mathrm{C}$ 下反应 $24 \mathrm{~h}$ 后, 冷至 室温, 抽干溶剂. 将粗产品溶于 $\mathrm{CDCl}_{3}$ 中进行 ${ }^{1} \mathrm{H} \mathrm{NMR}$ 测试以确定反应转化率. 将粗产品溶于乙酸乙酯经滤膜 过滤后进行 GC-MS 分析来确定产物构型比例.

产物分离: 待反应完成后, 旋干反应液, 通过柱层 析分离得到产物.

\section{References}

[1] (a) Trinquier, G. J. Am. Chem. Soc. 1990, 112, 2130. (b) Apeloig, Y.; Pauncz, R.; Miriam, K.; West, R. Steiner, W.; Chapman, D. Organometallics 2003, 22, 3250. (c) Sasamori, T.; Tokitoh, N. In Encyclopedia of Inorganic Chemistry II, Ed.: King, R. B., John 
Wiley \& Sons: Chichester, U.K., 2005, p. 1698.

[2] For selected papers: (a) Blom, B.; Stoelzel, M.; Driess. M. Chem. Eur. J. 2013, 19, 40. (b) Blom, B.; Gallego, D.; Driess, M. Inorg. Chem. Front. 2014, 1, 134. (c) Zhou, Y.-P.; Driess. M. Angew. Chem. Int. Ed. 2019, 58, 3715. (d) Raoufmoghaddam, S.; Zhou, Y. P.; Wang, Y.; Driess, M. J. Organomet. Chem. 2017, 829, 2. (e) Haaf, M.; Schmedake, T. A.; West, R. Acc. Chem. Res. 2000, 33, 704. (f) Yao, S.; Xiong, Y.; Driess, M. Organometallics 2011, 30, 1748. (g) Cui, H.; Teng, P.; Zhang, E.; Lu, J.; Zhang, F.; Wu, M. Chin. J. Chem. 2017, 35, 401. (h) Cui, H.; Cui, C. Chin. J. Org. Chem. 2016, 36, 626 (in Chinese). (崔海燕, 崔春明, 有机化学, 2016, 36, 626.)

[3] (a) Jutzi, P.; Kanne, D.; Krfiger, C. Angew. Chem. Int. Ed. 1986, 25 , 164. (b) Blom, B.; Stoelzel, M.; Driess. M. Chem. Eur. J. 2013, 19, 40. (c) Zhou, L.; Li, Y.; Lin, F.; Tian, D.; Lei, Q.; Fang, W.; Xie, H. Chin. J. Org. Chem. 2015, 35, 698 (in Chinese). (周莉, 李阳, 林芙 蓉, 田迪英, 雷群芳, 方文军, 谢湖均, 有机化学, 2015, 35, 698.) (d) Wang, L.; Guo, J.; Li, Y.; Su, Y.; Liu, J.; Li, Y.; Wang, S.; Shimada, S.; Huang, W. Chin. J. Chem. 2017, 35, 507.

[4] (a) Troadec, T.; Prades, A.; Rodriguez, R.; Mirgalet, R.; Baceiredo, A.; Saffon-Merceron, N.; Branchadell, V.; Kato, T. Inorg. Chem. 2016, 55, 8234. (b) Iimura, T.; Akasaka, N.; Iwamoto, T. Organometallics 2016, 35, 4071. (c) Iimura, T.; Akasaka, N.; Kosai, T.; Iwamoto, T. Dalton Trans. 2017, 46, 8868.

[5] (a) Cabeza, J. A.; García-Álvarez, P.; González-Álvarez, L. Chem. Commun. 2017, 53, 10275. (b) Ren, H.; Zhou, Y.-P.; Bai, Y.; Cui, C.; Driess, M. Chem. Eur. J. 2017, 23, 5663. (c) Brück, A.; Gallego, D.; Wang, W.; Irran, E.; Driess, M.; Hartwig, J. F. Angew. Chem. Int. Ed. 2012, 51, 11478. (d) Zhou, Y.-P.; Raoufmoghaddam, S.; Szilvási, T.; Driess, M. Angew. Chem. Int. Ed. 2016, 55, 12868. (e) Wang, Y.; Kostenko, A.; Yao, S.; Driess, M. J. Am. Chem. Soc. 2017, 139, 13499.
[6] (a) Fürstner, A.; Krause, H.; Lehmann, C. W. Chem. Commun. 2001, 2372. (b) Khoo, S.; Cao, J.; Yang, M.-C.; Shan, Y.-L.; Su, M.-D.; So, C.-W. Chem. Eur. J. 2018, 24, 14329. (c) Zhang, M.; Liu, X.; Shi, C.; Ren, C.; Ding, Y.; Roesky, H. W. Z. Anorg. Allg. Chem. 2008, 634, 1755. (d) Gallego, D.; Brgck, A.; Irran, E.; Meier, F.; Kaupp, M.; Driess, M.; Hartwig, J. F. J. Am. Chem. Soc. 2013, 135, 15617. (e) Tan, G.; Enthaler, S.; Inoue, S.; Blom, B.; Driess, M. Angew. Chem. Int. Ed. 2015, 54, 2214. (f) Qi, X.; Sun, H.; Li, X.; Fuhr, O.; Fenske, D. Dalton Trans. 2018, 47, 2581. (g) Mo, Z.; Kostenko, A.; Zhou, Y.-P.; Yao, S.; Driess, M. Chem. Eur. J. 2018, 24, 14608. (h) Schmidt, M.; Blom, B.; Szilvási, T.; Schomäcker, R.; Driess, M. Eur J. Inorg. Chem. 2017, 1284. (i) Someya, C. I.; Haberberger, M.; Wang, W.; Enthaler, S.; Inoue, S. Chem. Lett. 2013, 42, 286.

[7] (a) Bracher, F.; Litz, T.; J. Prakt. Chem./Chem.-Ztg. 1996, 338, 386 (b) Brown, H. C.; Chen, J. J. Org. Chem. 1981, 46, 3978. (c) Brown, H. C.; Rao, B. S. J. Am. Chem. Soc. 1959, 81, 6423. (d) Crockett, M. P.; Tyrol, C. C.; Wong, A. S.; Li, B.; Byers, J. A. Org. Lett. 2018, 20 5233. (e) Hartwig, J. F. Acc. Chem. Res. 2011, 45, 864. (e) Martin, R.; Buchwald, S. L. Acc. Chem. Res. 2008, 41, 1461. (f) Miyaura, N.; Suzuki, A. Chem. Rev. 1995, 95, 2457.

[8] Haberberger, M.; Enthaler, S. Chem. Asian J. 2013, 8, 50.

[9] Greenhalgh, M. D.; Thomas, S. P. Chem. Commun. 2013, 49, 11230.

[10] Nakajima, K.; Kato, T.; Nishibayashi, Y. Org. Lett. 2017, 19, 4323.

[11] Singh, A.; Shafiei-Haghighi, S.; Smith, C. R.; Unruh, D. K.; Findlater, M. Asian J. Org. Chem. 2020, 9, 416.

[12] Bai, Y.; Zhang, J.; Cui, C. Chem. Commun. 2018, 54, 8124

[13] (a) Docherty, J. H.; Peng, J.; Dominey, A. P.; Thomas, S. P. Nat. Chem. 2017, 9, 595. (b) Gorgas, N.; Alves, L. G.; Stöger, B.; Martins, A. M.; Veiros, L. F.; Kirchner, K. J. Am. Chem. Soc. 2017, 139,8130 . 